architecture in which connections between layers of simulated neurons are strengthened on the basis of experience. Deep-learning systems can then draw complex information from reams of unstructured data (see Nature 505, 146-148; 2014). Google, of Mountain View, California, uses such algorithms to automatically classify photographs and aims to use them for machine translation.

The second is reinforcement learning, a decision-making system inspired by the neurotransmitter dopamine reward system in the animal brain. Using only the screen's pixels and game score as input, the algorithm learned by trial and error which actions - such as go left, go right or fire - to take at any given time to bring the greatest rewards. After spending several hours on each game, it mastered a range of arcade classics, including car racing, boxing and Space Invaders.

Companies such as Google have an immediate business interest in improving AI, says Sutton. Applications could include how to best place advertisements online or how to prioritize

stories in news aggregators, he says. Sprague, meanwhile, suggests that the technique could enable robots to solve problems by interacting with their environments.

But a major driver is science itself, says Hassabis, because building smarter systems means gaining a greater understanding of intelligence. Many in computational neuroscience agree. Sprague, who has created his own version of DeepMind's algo-

"The tricks we use for training a system might lead to new ideas about the brain."

rithm, explains that whereas AI is largely irrelevant to neuroscience at the level of anatomical connections among neurons, it can bring insight at the higher level of computational principles.

Computer scientist Ilya Kuzovkin at the University of Tartu in Estonia, who is part of a team that has been reverse-engineering DeepMind's code since 2013, says: "The tricks we use for training a system are not biologically realistic. But comparing the two might lead to new ideas about the brain." A particular boost is likely to come from the DeepMind team's choice to publish its code alongside its research, Kuzovkin says, because his lab and others can now build on top of the result. "It also shows that industryfinanced research goes the right way: they share with academia," he adds.

DeepMind was bought by Google in 2014 for a reported $£ 400$ million (US $\$ 617$ million), and has been poaching leading computer scientists and neuroscientists from academia, growing from 80 to 140 researchers so far.

Its next steps are again likely to be influenced by neuroscience. One project could be building a memory into its algorithm, allowing the system to transfer its learning to new tasks. Unlike humans, when the current system masters one game, it is no better at tackling the next.

Another challenge is to mimic the brain's way of breaking problems down into smaller tasks. Currently, DeepMind's system struggles to link actions with distant consequences - a limitation that, for example, prevented it from mastering maze games such as Ms. Pac-Man.

\title{
Researchers seek definition of head-trauma disorder
}

\section{Guidelines should assist in diagnosis of brain disease seen in retired American footballers.}

\section{BY HELEN SHEN}

$\mathrm{D}$ ave Duerson suspected that something was wrong with his brain. By 2011, 18 years after the former American football player had retired from the Phoenix Cardinals, he experienced frequent headaches, memory problems and an increasingly short temper. Before he killed himself, he asked that his brain be donated for study.

Researchers who examined it found signs of chronic traumatic encephalopathy (CTE), a degenerative condition linked to repeated head injuries. At least 69 cases have been reported in the literature since 2000, many in former boxers and American football players (P. H. Montenigro et al. Alz. Res. Ther. 6, 68; 2014) - heightening public concern about concussions during contact sports. Yet much about CTE is unknown, from its frequency to its precise risk factors and even whether its pathology is unique.

Researchers now hope to take a major step towards answering those questions. At Boston University in Massachusetts on 25-27 February, neuroscientists will convene to examine the characteristics of CTE in brain tissue from post-mortem examinations. They hope to agree on a set of diagnostic criteria for the disease, and to assess whether it is distinct from other brain disorders, such as Alzheimer's disease.

The effort is sorely needed, says Walter Koroshetz, acting director of the US National Institute of Neurological Disorders and Stroke in Bethesda, Maryland, which is organizing the meeting. "The definition is the important piece that lets you do the rest of the research," he says. And the stakes are high. CTE is associated with memory loss, irritability, depression and explosive anger, which are thought to appear and worsen years after repeated head trauma. Research by Ann McKee, a neuropathologist

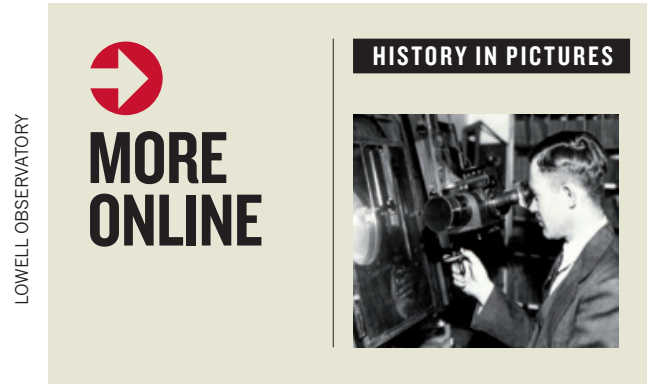

\begin{tabular}{l|l} 
& MORE NEWS \\
$\begin{array}{ll}\text { A celebration } \\
\text { of the 85th }\end{array}$ & $\begin{array}{l}\text { — Low leaks from US gas } \\
\text { fields go.nature.com/8cmvu6 }\end{array}$ \\
anniversary & — FDA ponders rules on DNA \\
of Pluto's & testing g0.nature.com/3ncdbh \\
discovery & @ Star skirted Solar System \\
go.nature.com/ & 70,000 years ago \\
vil2ci & go.nature.com/d6kxb6
\end{tabular}

VIDEO OF THE WEEK

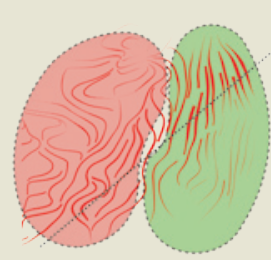

How countries 'flow' towards development go.nature. com/8hjslu 
